\title{
Venezia, Biblioteca Nazionale Marciana, gr. Z. 323 (coll. 630)
}

\author{
I. -III.-V.-VII. inizio del secolo XV; II.-IV.-VI. post 1368.
}

Comp.; ff. I-II, 1-487, III-IV; sul f. I è incollato un foglio, tratto dal catalogo di Zanetti (1741), con il contenuto del codice; sul f. 1r vi è l'ex-libris di Bessarione; num. moderna a penna nel marg. sup. il numero 5 è ripetuto due volte; il numero 345 manca. Sono bianchi i ff. 23-24, 38-40, 70v, 170, 204v, 207v, 212v-213, 227r, 250r-257v, 263v-265v, 290v, 303v, 308v, 383, 400v-402v, 461v, 476v-478. Legatura moderna in pelle bianca risalente alla campagna del 1722.

Manoscritto complesso, formato dall'alternarsi di sezioni riferibili alla seconda metà del secolo XIV (II, IV, VI) e di sezioni riconducibili al principio del secolo successivo (I, III, V, VII), realizzate con l'intento di completare o ampliare le prime con ulteriori testi di carattere astronomico.

\section{I. ff. 1-170 (Costantinopoli, inizio XV sec.)}

Comp.; fil. ff. 4-6 simile a tour (Br nr. 15864, 1419-1436), ff. 10, 11, 14, 15, 27, 30, 34 simile a fleur de lis (Br nr. 7271, 1408-1427), ff. 18, 20, 21, 23, 25, 32, 42, 44, 45, 50, 52, 53, 55, 57, 58, 63, 66, 68, 71, 73, $76,78,79,81,84,86,87,89,93,94,95,96,104,105$ simile a lettre $R$ (Br nr. 8934, 1405-1411), ff. 133, 139, 141, 145, 146, 147, 149, 150, 153, 165, 167 simile a hache (Br nr. 7514, 1383-1404), ff. 110, 111, 120, 125, 139 simile a monts ( $\mathrm{Br} \mathrm{nr} .11689,1411-1416)$, ff. 161, 162, 163 simile a tête de bouc (Br nr. 15490, 1389-1469); $1-8^{8}(64), 9^{10}(74), 10-21^{8}(170)$; num. fasc. assente; mm $208 \times 147=23 / / 155 / / 30 \times$ 11 // 6 // 91 // 10 // 6 // 22; a piena pagina, 1l. 27/rr. 29, unità di rigatura mm 5; rigatura tracciata a secco sul verso di ogni foglio, tipo S/L 30D1 (Muz 2-21/0/0/J) (f. 67).

\section{Scrittura}

Nell'unità si individua una sola mano: Copista AG (ff. 1r-22v, 25r-37v, 41r-70r, 71r-169v), mano individuale di XV secolo.

\section{Decorazione}

Rubriche in inchiostro rosso. 


\section{Contenuto}

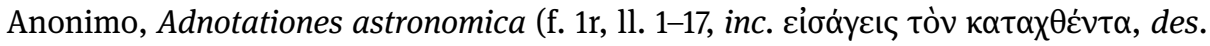

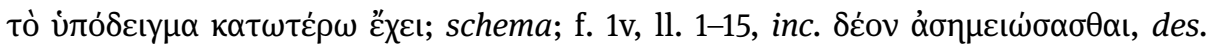

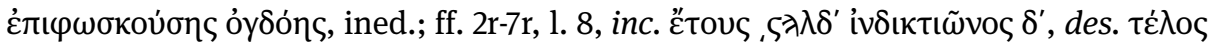

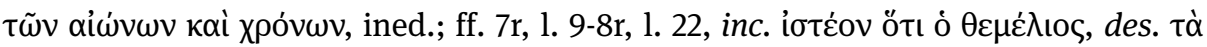

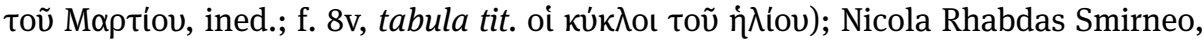

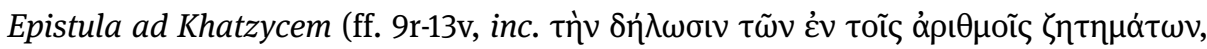

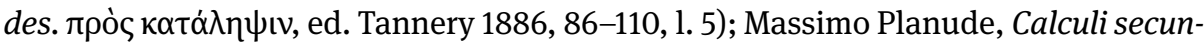

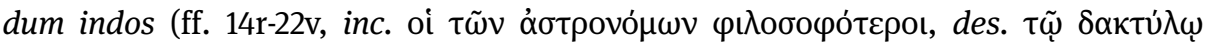

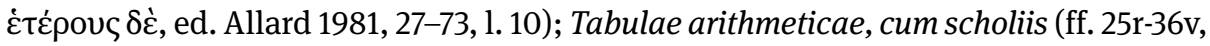

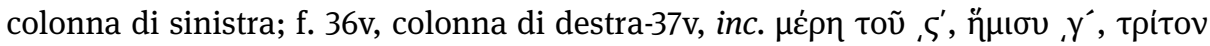

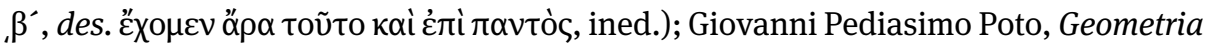

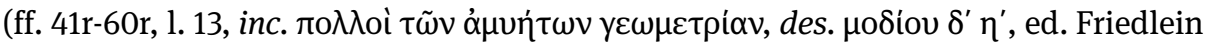

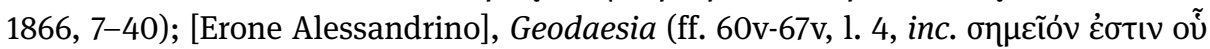

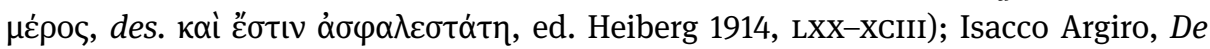

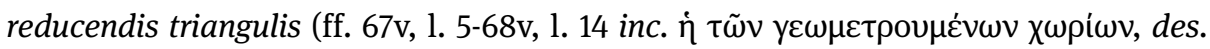

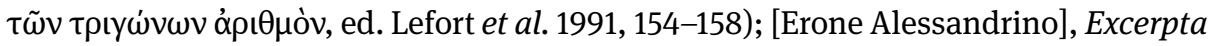

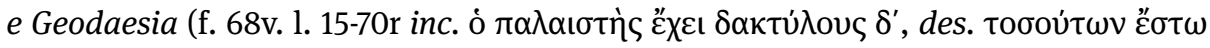
$\pi о \delta \tilde{\omega} \nu \dot{\eta} \delta$ เ $\alpha \dot{\mu} \varepsilon \rho \rho \varsigma$, ined.); Anonimo, Expositio in tabulas persicas (ff. 71r-94v, 1. 18,

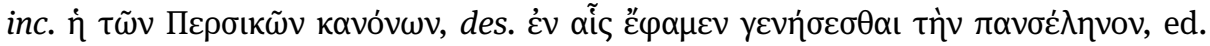
Bardi 2017); Tabulae astronomicae persicae (ff. 95r-165v, ined.); Anonimo, Praeparatio

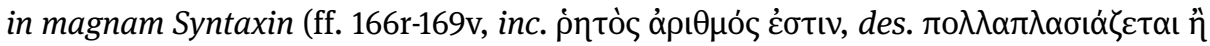
$\pi \mathrm{o} \lambda \lambda \alpha \pi \lambda \alpha \sigma \iota \alpha \dot{\zeta} \varepsilon \mathrm{l}$, ed. Moll 1965).

\section{171-210 (Costantinopoli, post 1368)}

Cart.; fil. ff. 177, 182, 183, 184, 188 simile a cercle (M/Tr nr. 2068, 1381), ff. 190, 191, 193, 196, 197, 200, 201, 203, 204 simile a saucisson (M/Tr nr. 6958, 1345-1350); 22-26 (210); num. fasc. presente nel marg. inf. est. del recto del primo foglio dei primi quattro fascicoli, numerati da $\alpha^{\prime}$ a $\delta^{\prime} ; \mathrm{mm}$ $207 \times 148$ = 20 // 149 // $36 \times 15 / / 97$ // 8 // 12 // 13; a piena pagina, ll. 31, unità di rigatura mm 4; rigatura tracciata a secco, tipo S/L 10D1 (Muz 1-11/0/0/J), sistema S/L 1 (f. 178).

\section{Scrittura}

Nell'unità sono intervenuti due copisti: Anonimo $B$ (ff. 171r-204r, 205r-207r, 1. 25, 208r210r); <Isacco Argiro (Mondrain 2007 [2008], 166; Bianconi 2008, 358)> (f. 210v). 


\section{Decorazione}

Fascia introduttiva, titolo e iniziale minore in ekthesis in rosso carminio.

\section{Contenuto}

Proclo Diadoco, Hypotyposis astronomicarum positionum (ff. 171r-204r, 1. 25, inc.

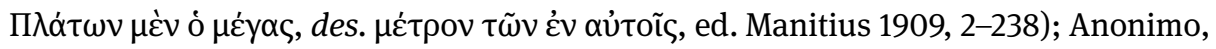

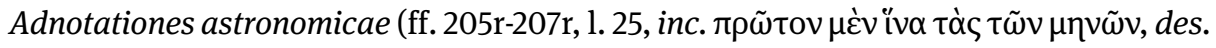

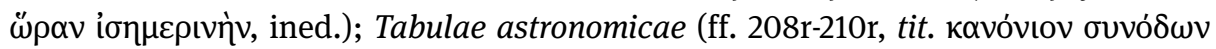

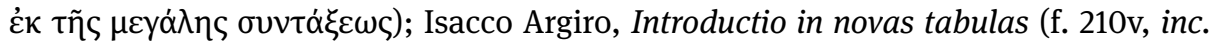

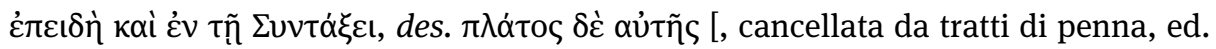
Wampach 1978-1979).

\section{III. ff. 211-214 (Costantinopoli, inizio XV sec.)}

Cart.; fil. assente; $27^{3+1}$ (214); num. fasc. assente; $\mathrm{mm} \mathrm{206 \times 147=10//16//140//} 40 \times 16 / / 90 / / 10$ // 6 // 25; a piena pagina, ll. 25/rr. 30, unità di rigatura mm 5; rigatura tracciata a secco sul verso di ogni foglio, tipo S/L 30D1 (Muz 2-21/0/0/J) (f. 211).

\section{Scrittura}

Nell'unità è presente un solo scriba: Copista AG (ff. 211r-212r, 1. 3, 214rv).

\section{Decorazione}

Rubriche in rosso.

\section{Contenuto}

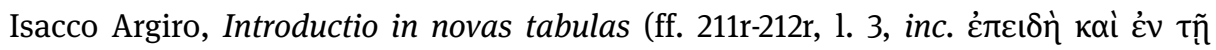

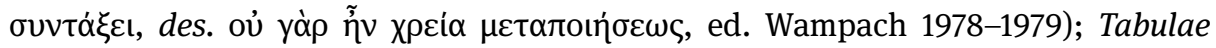

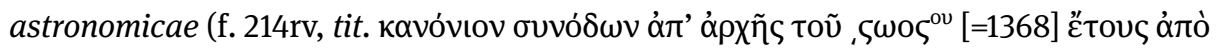

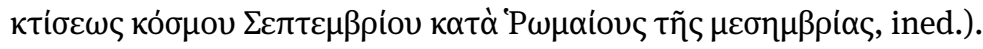




\section{IV. ff. 215-221 (Costantinopoli, post 1368)}

Cart.; fil. non riscontrata; $28^{6+1}$ (221); num. fasc. assente; $\mathrm{mm} 206 \times 147=20$ // 96 // 7 // $8 \times 27$ // 141 // 17 // 23; a piena pagina, ll. 28/rr. 28, unità di rigatura mm 5; rigatura tracciata a secco sul recto di alcuni fogli, tipo S/L 10D1 (Muz 1-11/0/0/J) (f. 225).

\section{Scrittura}

La sezione è stata vergata dagli stessi scribi che hanno collaborato alla realizzazione della seconda unità: Anonimo $B$ (ff. 217v, 218rv, 220r-220v, 1. 16) e <Isacco Argiro (ff. 215r-215v, 1. 18, 216rv, 220v, 1. 17-221v, 1. 4) (Mondrain 2007 [2008], 166; Bianconi 2008, 358)>.

\section{Decorazione}

Le iniziali minori e i titoli vergati in rosso sono di mano dei copisti, come anche i digrammi che corredano il testo (f. 218r vi digramma sul ciclo solare).

\section{Contenuto}

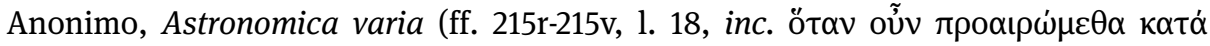

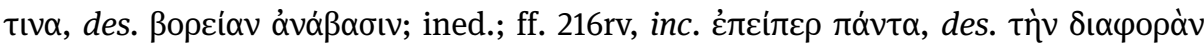

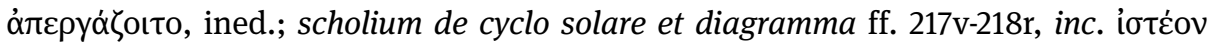

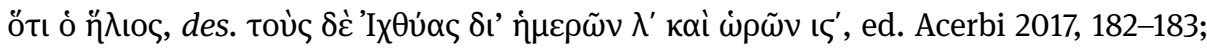

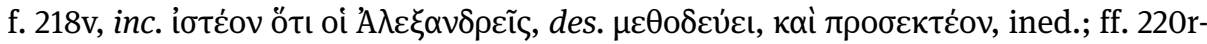

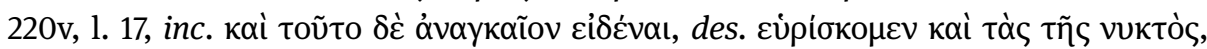

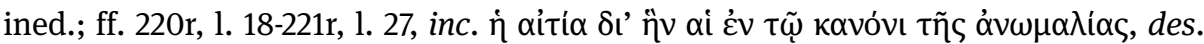

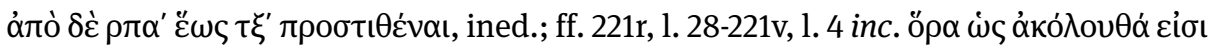

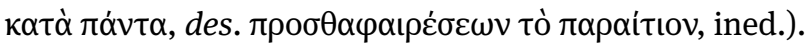

\section{V. ff. 222-265 (Costantinopoli, inizio XV sec.)}

Cart.; in quarto; fil. ff. 227, 228, 231, 232, 234, 237, 241 simile a $\operatorname{arc}$ (Br nr. 799, 1406-1411), ff. 244, 247 simile a lettere ( $\mathrm{Br}$ nr. 8934, 1405-1411); $29^{4}$ (225); 30-34 ${ }^{8}$ (265); num. fasc. assente; mm $206 \times$ 147 = 10 // 16 // 140 // $40 \times 16$ // 90 // 10 // 6 // 25; a piena pagina, ll. 29/rr. 32, unità di rigatura $\mathrm{mm}$ 5; rigatura tracciata a secco sul verso di alcuni fogli, tipo S/L 30D1 (Muz 2-21/0/0/J) (f. 258). 


\title{
Scrittura
}

L'unità si deve al copista della prima sezione: Copista AG (ff. 222r-225v, 1. 17, 226r-244v, 1. 22, 245r-249v, 258r-263r, 1. 11).

\section{Decorazione}

Rubriche in inchiostro rosso.

\section{Contenuto}

Stefano Alessandrino, In Tabulas manuales Ptolemaei capita 1-30 (ff. 222r-249v,

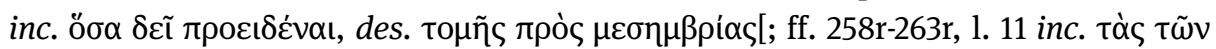

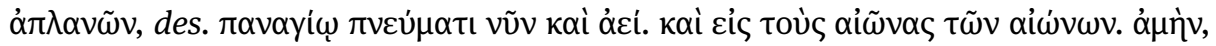
ed. [limitata ai capp. 1-16] Lempire 2016, 80-278).

\section{VI. ff. 266-478 (Costantinopoli, post 1368)}

\begin{abstract}
Cart.; in quarto, fil. ff. 266, 270, 275, 277, 285, 286, 290, 291, 303, 308, 364, 386, 387, 468 simile a cercle (M/Tr nr. 2068, 1381), ff. 288, 471, 472 simile a fruit poire (M/Tr nr. 4420, 1379), ff. 294, 296, 297, 299, 309 simile a licorne (M/Tr nr. 5827, 1365), ff. 395, 400, 413, 414, 422, 424, 429, 431, 437, 439, 447, 449, 461, 463 simile a arbalète $\left(\mathrm{M} / \mathrm{Tr} \mathrm{nr}\right.$. 298, 1354); $35-36^{8}(281), 37^{12}(293), 38^{6+1}(300), 39^{10}$ (310), 40-49 $(391), 50^{12-1}(402), 51-58^{8}(466), 59^{4}(470), 60^{8}(478)$; num. fasc. visibile nel marg. inf. est. del recto del primo foglio di alcuni fogli: $\gamma^{\prime}$ (f. 282), $\delta^{\prime}-\alpha^{\prime}$ (f. 319-376), $\gamma^{\prime}-\eta^{\prime}$ (ff. 419-459); mm $206 \times 147=27$ // 141 // 17 // $23 \times 20$ // 96 // 17 // 8; a piena pagina, ll. 29/rr. 29, unità di rigatura $\mathrm{mm}$ 5; rigatura tracciata a secco sul recto di alcuni fogli, tipo S/L 10D1 (Muz 1-11/0/0/J) (f. 410).
\end{abstract}

\section{Scrittura}

L'unità si deve agli stessi copisti che hanno realizzato la seconda e la quarta sezione: Anonimo B (ff. 266r-285r, 1. 12, 289r-303v, 304r-305r, 1l. 1-6, 305v-308r, 309r-343r, 1. 16, 343v-382v, 384r-393r, 1. 25, 403r-467v, 469r-470v, 1. 8) e < Isacco Argiro (Mondrain 2007 [2008], 167; Bianconi 2008, 358)>. Quest'ultimo ha copiato in alcuni fogli il testo principale (ff. 285r, 1. 14-288v, 1. 16, 394r-398v, 1. 13, 400r, 468rv, 471r-476r, 1. 19) e in altri le note a margine (ff. 267r, 270r, 272v, 273v, 274rv, 275rv, 276r, 276v, 277r, 277v, 278rv, 279rv, 280rv, 281rv, 305r, 1l. 6-21, 305v-308r, 310rv, 343r, 1. 17-343v, 344rv, 347350v, 363rv, 371r, 382r, 388v-389r, 412r, 438v-439r, 453rv, 460v, 462v-463r, 464r, 465rv, 466rv). 


\section{Decorazione}

Rubriche, schemi e diagrammi (ff. 291-382, 398v, 393v, 399r, 423v) in inchiostro rosso.

\section{Contenuto}

Teone Alessandrino, Commentarius parvus in Ptolemaei Tabulae manuales (ff. 276r-

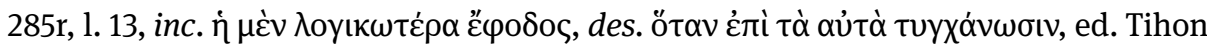
1978, 199-298); Additamenta ad praecedens opus (ff. 285r, 1. 14-286v, 1. 29, inc. ïva $\delta \dot{\varepsilon}$

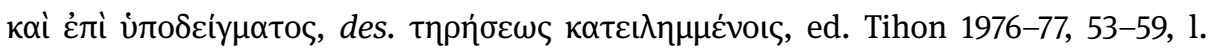

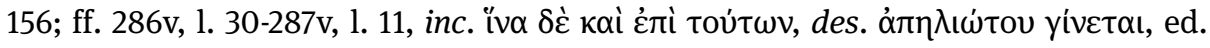
Tihon 1976-77, 59, 1. 157-61); Isacco Argiro, Introductio in novas tabulas (ff. 287v, 1.

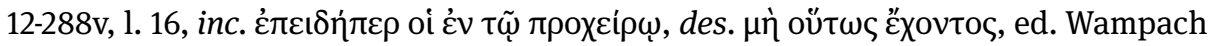
1978-1979); Claudio Tolomeo, Tabulae manuales (ff. 289r-303r, 304r-307v, 309-382r, ed. parz. Tihon 2011); Anonimo, Adnotatio astronomica (f. 382v, inc. ò $\mu$ غ̇v Kpóvos,

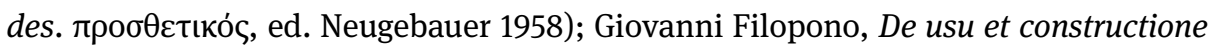

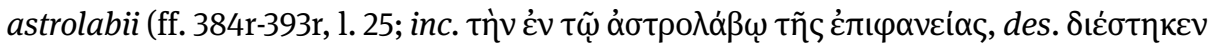

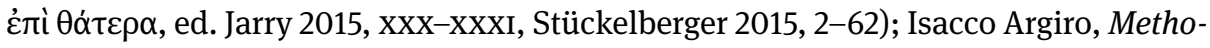

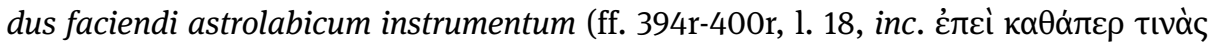

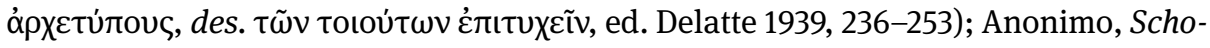

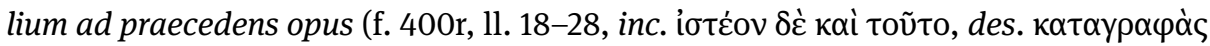

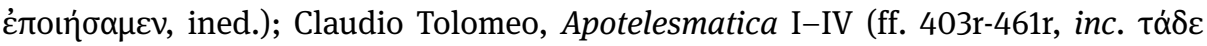

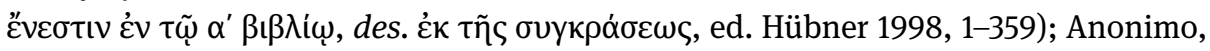
Tractatus astronomicus de iis quae vocantur centra (ff. 462r-466v, 1. 24, inc. Toũ $\zeta \omega \delta\llcorner\alpha \kappa о \tilde{~ \kappa u ́ \kappa \lambda o v, ~ d e s . ~ \tau \varepsilon ́ \lambda o \zeta ~ \varepsilon ́ \rho \chi o ́ ~} \mu \varepsilon \theta \alpha$, ined.); Claudio Tolomeo, Fructus (ff. 467r-

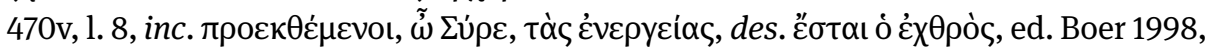

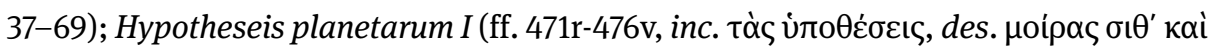

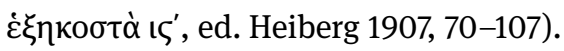

\section{VII. ff. 479-487 (Costantinopoli, inizio XV sec.)}

Cart.; fil f. 480 simile a hache (Br nr. 7514, 1383-1404), f. 487 simile a fleur (Br nr. 6687, 1391-1404); $161^{10-1}$ (487); num. fasc. assente; $\mathrm{mm} 206 \times 147=10 / / 16 / / 140 / / 40 \times 16 / / 90 / / 10 / / 6 / / 25 ; \mathrm{a}$ piena pagina, $11.26 / \mathrm{rr}$. 32 , unità di rigatura $\mathrm{mm} 5$; rigatura tracciata a secco sul verso di alcuni fogli, tipo S/L 30D1 (= 2-21/0/0/J Muz) (f. 485). 


\section{Scrittura}

L'unità è stata vergata da un solo copista: Copista AG (ff. 479r-487r, 1. 14, 487v).

\section{Decorazione}

Rubriche in inchiostro rosso con schemi e lettere delle figure.

\section{Contenuto}

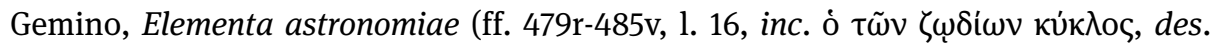

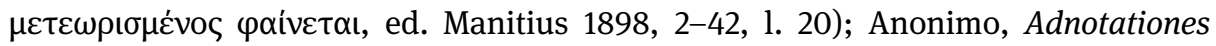

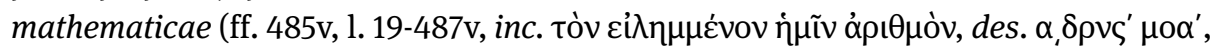
ed. f. 487rv Heiberg 1899, 172-173).

\section{Storia del codice}

Il manoscritto faceva parte della collezione libraria di Bessarione e figura con il nr. 230 nell'inventario del 1468, dove si registra la donazione della sua biblioteca alla basilica di San Marco a Venezia (Labowsky 1979, 166).

\section{Bibliografia}

\section{Cataloghi}

CCAG II, 2-4; Mioni (1985) 38-44.

\section{Edizioni}

Allard (1981); Acerbi (2017); Bardi (2017); Boer (1998); Delatte (1939); Friedlein (1866); Heiberg (1899); Heiberg (1907); Heiberg (1914); Hübner (1998); Jarry (2015); Lefort et alii (1991); Lempire (2016); Manitius (1898); Manitius (1909); Moll (1965); Neugebauer (1958); Stückelberger (2015); Tannery (1886); Tihon (1973); Tihon (1976-77); Tihon (1978); Tihon (2011); Wampach (1978-1979). 


\section{Codicologia e paleografia}

Bianconi (2008) 358; Mondrain (2007) [2008] 166; Lempire (2011) 241-266; Labowsky (1979) 166; Jarry (2009) 44-77.

\section{Riproduzioni}

Bianconi (2008) tav. 10. 